\title{
Proton pump inhibitors, fracture risk and selection bias: three studies, same database, two answers
}

\author{
F. de Vries • T.-P. van Staa $・ H$. G. M. Leufkens
}

Received: 3 June 2010 / Accepted: 4 June 2010 / Published online: 19 June 2010

(C) The Author(s) 2010. This article is published with open access at Springerlink.com

\section{Dear Editor,}

Two studies in 2000 and 2001, both conducted using the UK General Practice Research Database (GPRD), reported conflicting results on the potential beneficial effects of statin use and fracture risk. An extensive reanalysis of the results showed that selection bias in one study largely explained the discrepant findings and that the results did not support a hypothesis of beneficial effects on bone. The reanalysis showed that the risk of hip fractures was halved almost instantly after starting statins and waned thereafter, which is difficult to reconcile with a bone effect. The biological mechanism assumed in 2000 was that statins affected the mevolanate pathway as do the bisphosphonates. Rather than emphasising the summary relative risks (RRs) in the original statin analyses, the absence of a durable response should have limited the interpretation of the findings since the data

\footnotetext{
F. de Vries • T.-P. van Staa $\cdot$ H. G. M. Leufkens

Utrecht Institute for Pharmaceutical Sciences,

Universiteit Utrecht,

Utrecht, Netherlands

F. de Vries $\cdot$ T.-P. van Staa

General Practice Research Database (GPRD),

Medicines and Healthcare Products Regulatory Agency,

London, UK

F. de Vries $(\triangle)$

Division of Pharmacoepidemiology and Clinical Pharmacology,

Utrecht Institute for Pharmaceutical Sciences,

Sorbonnelaan 16 ,

3584 CA Utrecht, Netherlands

e-mail: f.devries@uu.nl

F. de Vries

MRC Epidemiology Resource Centre,

Southampton General Hospital,

Southampton, UK
}

did not support a biological mechanism for statins to increase the quality or quantity of bone [1].

Does history repeat itself? On 25 May 2010, the Food and Drug Administration (FDA) decided to add a warning of a possible increased risk of fractures to the labelling of proton pump inhibitors (PPIs), drugs that are widely used for the treatment of gastroesophageal reflux disease [2]. This decision was based on the FDA's internal review of seven epidemiological studies, including two studies that used GPRD, but again with conflicting results [3, 4]. Two recently published papers were not included in this review, including a third GPRD study [5]. The FDA review showed that only few studies have evaluated the duration of any effect between use of PPIs and risk of fracture. The two recent studies in GPRD [5] and the Dutch PHARMO database (which has been published as an abstract since mid 2009, but which is now in press in Osteoporosis International) showed that the association between PPI use and fracture risk at various fracture sites was highest during the first year of treatment (a 1.3-fold increased risk of hip fracture), and then attenuated with prolonged use (with a 0.9 -fold increased risk of hip fracture in patients who had used PPIs for $>7$ years [6]). Similar to findings with statins and fracture risk, selection bias could explain this pattern of risk with duration of use [1]. These data are not supportive of a hypothesis that PPIs modify the quality or quantity of bone.

The FDA review considered that the biological mechanisms for an increased risk of fractures with PPIs are not known. Despite this, the FDA review concluded that the available data suggested a possible increased risk of fractures with PPI use. In our view, evidence for drug effects should not be used on an assessment of deviations of summary RRs from unity but rather on an assessment on whether specific hypotheses of biological mechanisms of drug effects are supported by evidence. Given the weak and 
conflicting evidences, not only from epidemiological studies, but also for a pharmacological effect of PPIs on bone mineral density in humans, we feel that the label change of PPIs is premature.

Conflicts of interest The Department of Pharmacoepidemiology and Clinical Pharmacology, Utrecht Institute for Pharmaceutical Sciences, has received unrestricted research funding from the Netherlands Organisation for Health Research and Development (ZonMW), the private-public funded Top Institute Pharma (www.tipharma.nl, includes co-funding from universities, government and industry), the EU Innovative Medicines Initiative, the Dutch Medicines Evaluation Board, the Dutch Ministry of Health and GlaxoSmithKline. GPRD is owned by the UK Department of Health and operates within the Medicines and Healthcare products Regulatory Agency (MHRA). GPRD is funded by the MHRA, Medical Research Council, various universities, contract research organisations and pharmaceutical companies. HGML is Chair of the Dutch Medicines Evaluation Board and co-opted member of the Committee for Medicinal Products for Human Use of the European Medicines Agency in London, United Kingdom. None of the views in this letter represent any of the official positions of any of these regulatory bodies.

Open Access This article is distributed under the terms of the Creative Commons Attribution Noncommercial License which per- mits any noncommercial use, distribution, and reproduction in any medium, provided the original author(s) and source are credited.

\section{References}

1. de Vries F, de Vries C, Cooper C, Leufkens B, van Staa T-P (2006) Reanalysis of two studies with contrasting results on the association between statin use and fracture risk: the General Practice Research Database. Int J Epidemiol 35:1301-1308

2. US Food and Drug Administration FDA (2010) Possible fracture risk with high dose, long-term use of proton pump inhibitors. http://www. fda.gov/Drugs/DrugSafety/PostmarketDrugSafetyInformationfor PatientsandProviders/ucm213206.htm. Accessed 28 May 2010

3. Yang YX, Lewis JD, Epstein S, Metz DC (2006) Long-term proton pump inhibitor therapy and risk of hip fracture. JAMA 296:29472953

4. Kaye JA, Jick H (2008) Proton pump inhibitor use and risk of hip fractures in patients without major risk factors. Pharmacotherapy 28:951-959

5. de Vries F, Cooper AL, Cockle SM, van Staa TP, Cooper C (2009) Fracture risk in patients receiving acid-suppressant medication alone and in combination with bisphosphonates. Osteoporos Int 20:1989-1998

6. Lalmohamed A, Pouwels S, Cooper C, van Staa TP, Leufkens B, de Boer A, de Vries F (2009) Use of proton pump inhibitors and risk of hip fracture. Bone 44(Suppl2):S396-S397 